\title{
A Set of Space-Time Block Codes for the High-Rate Transmission Scheme with One Information Bit
}

\author{
Yier Yan ${ }^{1,3}$, Xueqin Jiang ${ }^{2,3}$, Moon Ho Lee ${ }^{3}$ \\ ${ }^{1}$ School of Mechanical and Electrical Engineering of Guangzhou University, Guangdong Guangzhou, China \\ ${ }^{2}$ School of Information Science and Technology of DonghuaUniversity, Shanghai, PR China \\ ${ }^{3}$ Electrical and Computer Engineering of Chonbuk National University, Repulic of Korea \\ Email: year0080@gzhu.edu.cn, xqjiang@dhu.edu.cn, moonho@jbnu.ac.kr
}

Received 2013

\begin{abstract}
In [1], the authors have proposed one high rate transmission scheme for Space-Time Block Codes (STBC) without additional system source such as power, bandwidth and time slot. To maintain the full rank property of the coding gain matrix, we propose a set of STBCs for 4 transmit antennas transmission to transmit one additional information bit achieving rate-9/8. Another orthogonal STBC code with rate-1 is proposed in this paper within the set. It shows by computer simulation results that by employing the set of STBCs, it achieves better bit error rate (BER) performance and throughput than that of [1] with a valid BER improvement at the high SNR region above 20dB.
\end{abstract}

Keywords: Antennas; Orthogonal; STBC; BER; High Rate

\section{Introduction}

Since transmit diversity for wireless communication has been introduced in $[2,3]$. Space-time block coding (STBC) is an efficient transmit diversity scheme to combat detrimental effects of wireless fading channels. STBC from orthogonal designs are attracting wider attention due to their amenability for fast maximum likelihood (ML) decoding algorithm and full diversity [3,4]. STBCs are a set of practical signal design techniques aimed at approaching the information theoretic capacity for multiple-input and multiple-output (MIMO) channels. Alamouti code is an elegant and seminal STBC design for a two-transmit antenna system [4]. It achieves full-rate, i.e. rate one, full diversity transmission using two time slots for signals with complex constellations, which are employed in most current commercial wireless systems. The orthogonal code design from Tarokh et al. is a generalization of Alamouti code for systems with an arbitrary number of transmit antennas [2,3]. It has been proved however, the orthogonal design for complex signals with linear decoding complexity achieving full-rate full-diversity transmission is not available for the number of antennas more than two [3]. The system with higher number of antennas has to either suffer from rate loss or put up with more decoding complexity.

The class of linear STBC is the major category of space-time codes and can be divided into subclasses like Linear Dispersion Codes [5], Orthogonal STBC (OSTBC) [2-4] and Quasi Orthogonal STBC (QOSTBC) [6] which is typical designed for more than two antenna systems with increased, but not exponentially, decoding complexity [4-6].

A class of STBCs [1,7] is proposed for a high rate $>1$ transmission scheme by exploiting the inherent algebraic structure for 2 and 4 transmit antennas. In order to maintain the full rank property of coding gain matrix for two STBC codewords selected from two STBCs, respectively, one of two STBCs should be scaled by weighted factor and rotated by an angle. To compare the high rate transmission scheme with the rate- 1 case such as Alamouti and Jafarkhani schemes, the system performance is attenuated due to rotating or scaling one transmit matrix selected from the class. In order to compensate the loss of system performance, we propose another orthogonal STBC matrix with rate- 1 as one candidate in the set to maintain the full rank property of coding gain matrix for 4 transmit antennas case while two codewords are selected from two candidate matrices, respectively. Simulation results show that the BER performance is similar with Jafarkhani code in the high SNR region (20-25dB) due to full rank property of coding gain matrix.

\section{System Model}

Consider a MIMO system with $N$ transmitting and $M$ receiving antennas with transmit vector $\mathbf{x}=\left[x_{1}, \ldots, x_{N}\right]$ and flat block-fading channel [4] can be expressed as

$$
\mathbf{Y}=\mathbf{X H}+\mathbf{N}
$$




$$
\mathbf{Y}=\left[\mathbf{y}^{T}(1), \cdots, \mathbf{y}^{T}(T)\right]^{T}
$$

where

$$
\begin{aligned}
& \mathbf{X}=\left[\mathbf{x}^{T}(1), \cdots, \mathbf{x}^{T}(T)\right]^{T} \\
& \mathbf{N}=\left[\mathbf{n}^{T}(1), \cdots, \mathbf{n}^{T}(T)\right]^{T}
\end{aligned}
$$

are the matrices of the received signals, transmitted signals, and noise, respectively, $\mathbf{H}$ is the $N \times M$ complex channel matrix which is composed of i.i.d complex Gaussian entries, and $\mathbf{n}(t)$ is a vector of size $M$ representing the additive noise, $T$ is the block length. Here,

$$
\begin{aligned}
\mathbf{y}(t) & =\left[y_{1}(t), \cdots, y_{M}(t)\right], \quad \mathbf{x}(t)=\left[x_{1}(t), \cdots, x_{N}(t)\right], \\
\mathbf{n}(t) & =\left[n_{1}(t), \cdots, n_{M}(t)\right],
\end{aligned}
$$

are the complex row vectors of the received signal, transmitted signal, and noise, respectively.

\section{Original Works}

The author proposed one transmission scheme to transmit one additional information bit without additional source. The transmission scheme employs two STBC matrices $\mathcal{G}_{1}$ and $\mathcal{G}_{2}$ to represent the information of bit b for 0 or 1 , respectively

$$
\mathcal{G}_{1}\left(x_{1}, x_{2}, x_{3}, x_{4}, b=0\right)=\left(\begin{array}{cccc}
x_{1} & x_{2} & x_{3} & x_{4} \\
-x_{2}^{*} & x_{1}^{*} & -x_{4}^{*} & x_{3}^{*} \\
-x_{3}^{*} & -x_{4}^{*} & x_{1}^{*} & x_{2}^{*} \\
x_{4} & -x_{3} & -x_{2} & x_{1}
\end{array}\right),
$$

and

$$
\mathcal{G}_{2}\left(x_{1}, x_{2}, x_{3}, x_{4}, b=1\right)=\left(\begin{array}{cccc}
x_{1} e^{j \theta} & x_{2} e^{j \theta} & x_{3} & x_{4} \\
-x_{2}^{*} e^{j \theta} & x_{1}^{*} e^{j \theta} & -x_{4}^{*} & x_{3}^{*} \\
-x_{3}^{*} & -x_{4}^{*} & x_{1}^{*} e^{j \theta} & x_{2}^{*} e^{j \theta} \\
x_{4} & -x_{3} & -x_{2} e^{j \theta} & x_{1} e^{j \theta}
\end{array}\right),
$$

where each row represents an antenna index and each column represents either a time instance for STBC, and $x_{1}, x_{2}, x_{3}, x_{4}$ are selected from an alphabet (QPSK Modulation). Superscript * denotes complex conjugate. The system performance is mainly dependent on the coding gain matrix between $\mathcal{G}_{1}$ and $\mathcal{G}_{2}$. The coding gain defined in [2]

$$
C G=\left(C_{1}-C_{2}\right)\left(C_{1}-C_{2}\right)^{H}
$$

must be a full rank matrix with the determinant as large as possible, where $C_{1}$ and $C_{2}$ are STBC codewords [1] corresponding to the STBC matrix. It is easy to check the coding gain matrix for $\mathcal{G}_{1}$ and $\mathcal{G}_{2}$.

$$
C_{1}-C_{2}=\left(\begin{array}{cccc}
\hat{x}_{1} & \hat{x}_{2} & 0 & 0 \\
-\hat{x}_{2}^{*} & \hat{x}_{1}^{*} & 0 & 0 \\
0 & 0 & \hat{x}_{1}^{*} & \hat{x}_{2}^{*} \\
0 & 0 & -\hat{x}_{2} & \hat{x}_{1}
\end{array}\right)
$$

where $\hat{x}_{1}$ is $x_{1}\left(1-e^{j \theta}\right)$ and $\hat{x}_{2}$ is $x_{2}\left(1-e^{j \theta}\right)$.

Remarks: Although the coding gain matrix between $\mathcal{G}_{1}$ and $\mathcal{G}_{2}$ is one full rank matrix, the determinate would be different depending on the values of $e^{j \theta}$. The system performance is weak because there are many null parts in the coding matrix.

\section{Transmission Scheme}

To check previous works [1,7], the diversity gain of high rate transmission of STBCs is weak compared to Jafarkhani code with rate-1. The simulation results [1,7] show that the BER gap between [1] and Jafarkhani code [6] has an almost 3dB gap in the high SNR region (15-25dB). We propose a new set of STBCs in this paper to transmit one additional information bit b with an improvement of BER performance compared to [1].

\subsection{A Set of STBC Matrices}

Similar to the method of [1], we consider a set of $4 \times 4$ STBCs consisting of two STBC matrices $\mathcal{G}_{11}$ QOSTBC [6] and $\mathcal{G}_{22}$. Here, we propose $\mathcal{G}_{22}$ OSTBC as a candidate and use $\mathcal{G}_{11}$ for 4-antennas transmission scheme to transmit one additional information bit

$$
\begin{aligned}
& \mathcal{G}_{11}\left(x_{1}, x_{2}, x_{3}, x_{4}, b=0\right)=\left(\begin{array}{cccc}
x_{1} & x_{2} & x_{3} & x_{4} \\
-x_{2}^{*} & x_{1}^{*} & -x_{4}^{*} & x_{3}^{*} \\
-x_{3}^{*} & -x_{4}^{*} & x_{1}^{*} & x_{2}^{*} \\
x_{4} & -x_{3} & -x_{2} & x_{1}
\end{array}\right) . \\
& \mathcal{G}_{22}\left(x_{1}, x_{2}, x_{3}, x_{4}, b=1\right)=\left(\begin{array}{cc}
\mathcal{G}_{0}(y, z) & 0 \\
0 & \tilde{\mathcal{G}}_{0}(y, z)
\end{array}\right) .
\end{aligned}
$$

The $\mathcal{G}_{0}(y, z)$ is the Alamouti code [4] and shown as

$$
\begin{aligned}
& \mathcal{G}_{0}(y, z)=\left(\begin{array}{cc}
x_{1} e^{j \phi_{1}}+x_{2} & x_{3} e^{j \phi_{2}}+x_{4} \\
-\left(x_{3} e^{j \phi_{2}}+x_{4}\right)^{*} & \left(x_{1} e^{j \phi_{1}}+x_{2}\right)^{*}
\end{array}\right) \\
& \tilde{\mathcal{G}}_{0}(y, z)=\left(\begin{array}{cc}
\left(x_{1} e^{j \phi_{1}}+x_{2}\right)^{*} & \left(x_{3} e^{j \phi_{2}}+x_{4}\right)^{*} \\
-\left(x_{3} e^{j \phi_{2}}+x_{4}\right) & x_{1} e^{j \phi_{1}}+x_{2}
\end{array}\right)
\end{aligned}
$$

where we denote $\mathrm{y}$ as $x_{1} e^{j \phi_{1}}+x_{2}$ and denote $\mathrm{z}$ as $x_{3} e^{j \phi_{2}}+x_{4}$. It is easy to check the orthogonality and full rate property of $\mathcal{G}_{22}$

$$
\mathcal{G}_{22}^{H} \mathcal{G}_{22}=\left(\begin{array}{cccc}
|y|^{2}+|z|^{2} & 0 & 0 & 0 \\
0 & |y|^{2}+|z|^{2} & 0 & 0 \\
0 & 0 & |y|^{2}+|z|^{2} & 0 \\
0 & 0 & 0 & |y|^{2}+|z|^{2}
\end{array}\right) \text {, }
$$

where $\mid \cdot$ denotes absolute value and $\mathrm{H}$ denotes the 
Hermitian of a matrix.

\subsection{Angle Selections for $\phi_{1}$ and $\phi_{2}$}

To observe equation (11), denote $C_{1}$ as the first transmit codeword with signal $x_{1}, x_{2}, x_{3}, x_{4}$ and denote $C_{2}$ as the second transmit codeword with signal $\tilde{x}_{1}, \tilde{x}_{2}, \tilde{x}_{3}, \tilde{x}_{4}$ employing transmit matrix $\mathcal{G}_{22}$. The determinant of coding gain matrix for $C_{1}$ and $C_{2}$ is zero if $x_{1}=x_{2}$, $x_{3}=-x_{4}, \tilde{x}_{1}=\tilde{x}_{2}, \tilde{x}_{3}=-\tilde{x}_{4}$. Otherwise, the determinant is extremely large if $x_{1}=-x_{2}, x_{3}=x_{4}, \tilde{x}_{1}=-\tilde{x}_{2}, \tilde{x}_{3}=\tilde{x}_{4}$. In order to avoid this problem, the values of $\phi_{1}$ and $\phi_{2}$ should be constrained to make the $x_{1} e^{j \phi_{1}}+x_{2}$ and $x_{3} e^{j \phi_{2}}+x_{4}$ none zeros employing QPSK modulation. Then, $\phi_{1}$ and $\phi_{2}$ must satisfy $\phi_{1} \neq k \pi / 4$ and $\phi_{2} \neq k \pi / 4$, $k=0,1,2, \ldots, N$. The set of these two STBC codes $\mathcal{G}=\left\{\mathcal{G}_{11}, \mathcal{G}_{22}\right\}$ is used to transmit one additional bit with optimization of coding gain in [1] achieving high rate-9/8 (QPSK). The information of the additional bit b comes from these two candidate matrices. While the information of the additional bit $b$ is 0 the candidate (7) is selected as the transmitted matrix. Otherwise, the candidate (8) is selected as another transmitted matrix to transmit additional bit $b=1$. To achieve good diversity performance of STBCs in the set $\mathcal{G}$, the coding gain defined in [2] $C G=\left(C_{1}-C_{2}\right)$ $\left(C_{1}-C_{2}\right)^{H}$ must be a full rank matrix. The drawback in [1] is that while $C_{1}$ and $C_{2}$ are selected from $\mathcal{G}_{11}$ and $\mathcal{G}_{22}$, respectively, the coding gain matrix $C G$ is singular or null in submatrix to lose full rank property. To solve this problem in [1], the authors proposed a criterion to scale or rotate the transmitted symbols in $\mathcal{G}_{11}$ or $\mathcal{G}_{22}$, by a weighted factor and an angle to guarantee full rank property [2]. Due to the weighted factor and rotated angle the coding gain matrix also loses little diversity gain while the STBC multiplied with a weighted factor individually transmits to the receiver. In order to compensate the loss of diversity gain we also propose another orthogonal STBC matrix $\mathcal{G}_{22}$ to make the coding gain matrix be full rank. If this coding gain matrix without a weighted factor or null submatrices is also full rank, the design does not lose diversity gain.

\subsection{Analysis of Coding Gain Matrix}

In order to select the optimum values of $\phi_{1}$ and $\phi_{2}$, the determinate of the coding gain matrix should be as large as possible,

$$
\begin{gathered}
\max \left(C_{1}-C_{2}\right)\left(C_{1}-C_{2}\right)^{H} \\
\text { subject to } 0 \leq \phi_{1}, \phi_{2} \leq 2 \pi ; \phi_{1}, \phi_{2} \neq k \pi / 4,
\end{gathered}
$$

where $C_{1}$ and $C_{2}$ are selected from $\mathcal{G}_{11}$ and $\mathcal{G}_{22}$, respectively. We will neglect the mathematical derivation to solve the problem, but these values can be verified by computer simulation results. Then, these optimum values are $\phi_{1}=3 \pi / 8$ and $\phi_{2}=3 \pi / 8$, respectively. Assume that $C_{1}$ is selected from matrix $\mathcal{G}_{11}$ and $C_{2}$ is selected from $\mathcal{G}_{22}$ with same information symbols, the coding gain matrix is given by (13)

$$
\begin{aligned}
C G & =\left(\begin{array}{cccc}
x_{1}+\breve{x}_{1}-x_{2} & x_{2}-\left(\breve{x}_{3}+x_{4}\right) & \breve{x}_{3} & x_{4} \\
-x_{2}^{*}+\left(\breve{x}_{3}^{*}+x_{4}^{*}\right) & x_{1}^{*}+x_{1}^{*}-x_{2}^{*} & -x_{4}^{*} & \widetilde{x}_{3}^{*} \\
-\breve{x}_{3}^{*} & -x_{4}^{*} & x_{1}^{*}+x_{1}^{*}-x_{2}^{*} & x_{2}^{*}-\left(\widetilde{x}_{3}^{*}+x_{4}^{*}\right) \\
x_{4} & -\breve{x}_{3} & x_{2}-\left(\breve{x}_{3}+x_{4}\right) & x_{1}+\breve{x}_{1}-x_{2}
\end{array}\right)^{H} \\
& \times\left(\begin{array}{cccc}
x_{1}+\breve{x}_{1}-x_{2} & x_{2}-\left(\breve{x}_{3}+x_{4}\right) & \breve{x}_{3} & x_{4} \\
-x_{2}^{*}+\left(\widetilde{x}_{3}^{*}+x_{4}^{*}\right) & x_{1}^{*}+x_{1}^{*}-x_{2}^{*} & -x_{4}^{*} & \widetilde{x}_{3}^{*} \\
-\breve{x}_{3}^{*} & -x_{4}^{*} & x_{1}^{*}+x_{1}^{*}-x_{2}^{*} & x_{2}^{*}-\left(\widetilde{x}_{3}^{*}+x_{4}^{*}\right) \\
x_{4} & -\breve{x}_{3} & x_{2}-\left(\breve{x}_{3}+x_{4}\right) & x_{1}+\breve{x}_{1}-x_{2}
\end{array}\right)^{H}
\end{aligned}
$$

where $\breve{x}_{1}=x_{1} e^{j \phi_{1}}$ and $\breve{x}_{3}=x_{3} e^{j \phi_{2}}$. To simplify the equation (13), let $y_{1}$ denote $x_{1}+\breve{x}_{1}-x_{2}$ and $y_{2}$ denote $x_{2}-\left(\breve{x}_{3}+x_{4}\right)$. Obviously, this coding gain matrix (13) has the same form as (7),

$$
C_{1}-C_{2}=\left(\begin{array}{cccc}
y_{1} & y_{2} & x_{3} & x_{4} \\
-y_{2}^{*} & y_{1}^{*} & -x_{4}^{*} & x_{3}^{*} \\
-x_{3}^{*} & -x_{4}^{*} & y_{1}^{*} & y_{2}^{*} \\
x_{4} & -x_{3} & -y_{2} & y_{1}
\end{array}\right)
$$

and is similar to QOSTBC with full rank property. Assume that QPSK modulation is employed in the transmission, the terms of $x_{1}+\breve{x}_{1}-x_{2}$ and $x_{2}-\left(\breve{x}_{3}+x_{4}\right)$ are none zeros for all combinations of $x_{1}, x_{2}, x_{3}, x_{4}$. Furthermore, the coding gain matrix $C G=\left(C_{1}-C_{2}\right)$ $\left(C_{1}-C_{2}\right)^{H}$ maintains full rank without null submatrices for all $C_{1} \in \mathcal{G}_{11}$ and $C_{2} \in \mathcal{G}_{22}$ without loss of coding gain compared to [1]. Although the STBC matrix $\mathcal{G}_{22}$ involves the orthogonal matrix form, $\mathcal{G}_{22}$ does not achieve full diversity property. It can be shown from the simulation result that there is a small gap between the $\mathcal{G}_{11}$ and $\mathcal{G}_{22}$

\subsection{Decoding Algorithm}

Here, we consider the problem of calculating the metric for the system model (1)

$$
\min \|\mathbf{Y}-\mathbf{H X}\|^{2}
$$

for the implementation of a sequential decoding algorithm. The decoding algorithm can be divided into two sub-decoding procedures: 1) We select all possible sequences for $x_{1}, x_{2}, x_{3}, x_{4}$ from the first candidate relying on (7) to decode the transmitted symbols $\left\|\mathbf{Y}-\mathbf{H X}_{1}\right\|^{2}$, where $\mathbf{X}_{1}$ corresponds to the first candidate STBC $\mathcal{G}_{1}$; 2) Similarly, we also use this ML principle to decode other possible transmitted symbols selected from another candidate $\left\|\mathbf{Y}-\mathbf{H X}_{2}\right\|^{2}$, where $\mathbf{X}_{2}$ corresponds to the first candidate STBC $\mathcal{G}_{1}$; 3) To decode the additional information bit, we also have to compare these two metrics for decoding it 


$$
\left\|\mathbf{Y}-\mathbf{H X}_{1}\right\|_{\leq}^{2}\left\|\mathbf{Y}-\mathbf{H} \mathbf{X}_{2}\right\|^{2}
$$

The bit information is dependent on minimum metric between these two metrics substituting previous decoding results into first step.

\section{Numerical Results}

In this section, some simulation results demonstrate validity of the proposed transmission scheme. QPSK modulation and flat fading channel are considered in all simulations. Two combinations of $\phi_{1}$ and $\phi_{2}$ are shown to verify the BER performance depending on different values of $\phi_{1}$ and $\phi_{2}$ in Figure 1.

Figure 2 shows the BER performances versus SNR to compare the proposed design with the QOSTBC $\mathcal{G}_{1}$ (7) design [6] and conventional high rate design [1] equipped with 4 transmitting and receiving antennas. The system performance of the proposed OSTBC $\mathcal{G}_{22}$ (8) deserves a similar performance with $\mathcal{G}_{11}$ above $20 \mathrm{~dB}$. In the high SNR region $20-25 \mathrm{~dB}$ of $\mathcal{G}_{11}$, the BER performance of the proposed high rate- $9 / 8$ transmission scheme is similar to the QOSTBC case, but there also exists a visible gap in the low SNR region 10-20dB. The reason is that the system performance depends on $\mathcal{G}_{11}$, $\mathcal{G}_{22}$ and coding gain matrix between them, and the curve should deserve a similar form with $\mathcal{G}_{11}$ and $\mathcal{G}_{22}$. Moreover, the BER performance of proposed design is almost $2 \mathrm{~dB}$ away from the conventional high rate-9/8 design with power scaling or angle rotation [1] at the BER of $10^{-5}$.

In Figure 3, the effective throughput performance is shown to compare the proposed scheme and the original works [1]. The effective throughput is defined as $\mathcal{R}=$ $2 R(1-F E R)$, where $R$ is the space-time code rate [2] and FER means the frame error rate, and each frame

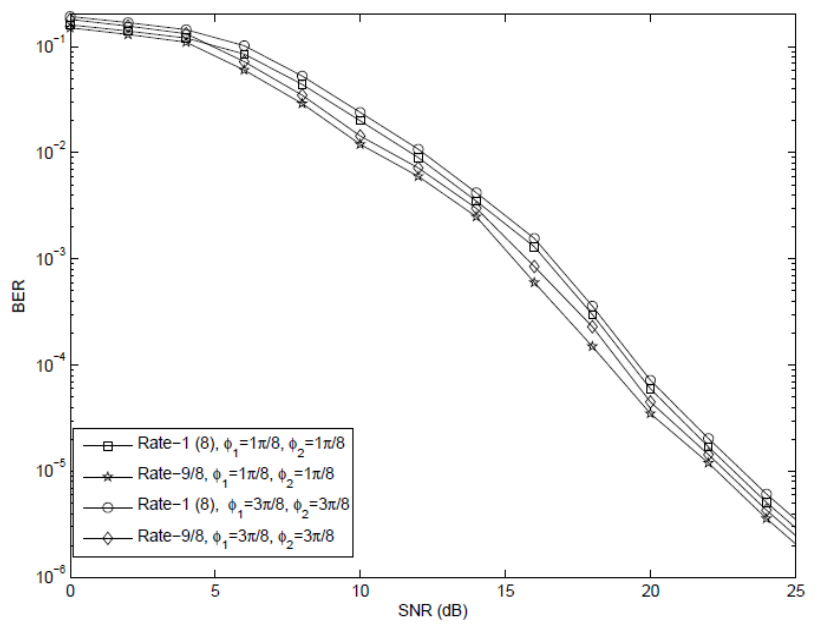

Figure 1. BER performance of high rate design with different angles.

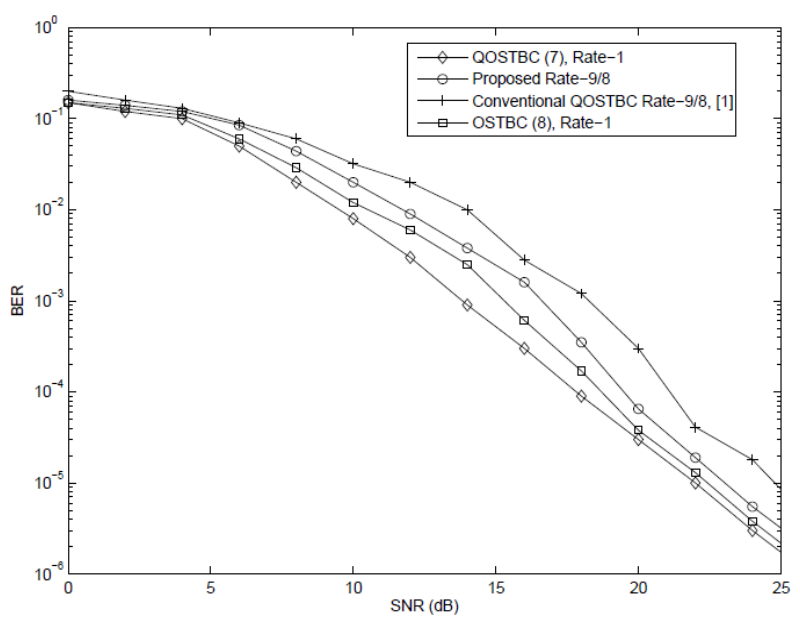

Figure 2. BER performance of high rate design for 4 antennas.

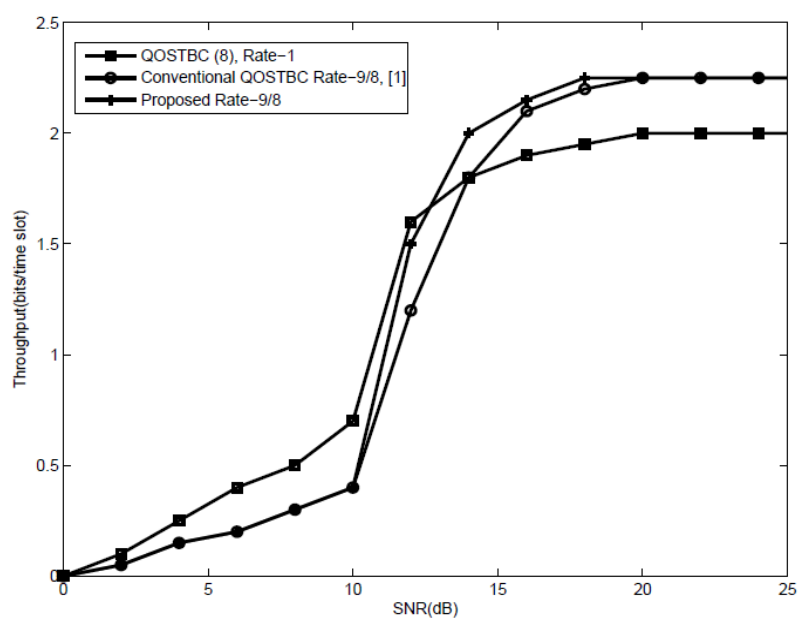

Figure 3. Effective throughputs of high rate design for 4 antennas.

contains 9 bits information. In the high SNR region $F E R \approx 0$ similar to Figure 2 that the \$FER\$ corresponds to BER and the BER performance of the proposed scheme is better than that of [1], the proposed transmission scheme achieves 2.25 bits per channel transmission whereas the effective throughput of the QOSTBC is 2 bits. A crossing point exits at a SNR level of $12 \mathrm{~dB}$. Similarly, the effective throughput performance of high rate-9/8 [1] is also simulated in this figure. From $10 \mathrm{~dB}$ to $20 \mathrm{~dB}$ of SNR, the effective throughput has been improved compared to [1] due to low FER in the proposed design.

\section{Conclusions}

A new set of STBCs including OSTBC and QOSTBC is proposed to transmit one additional information bit achieving high rate-9/8. The OSTBC is an candidate in the set to maintain the full rank property of the coding 
gain matrix. From the simulation results, the system performance is improved with a similar BER performance to Jafarkhani scheme in the high SNR region. In the future works, we are interested in enlarging the set of STBC matrices and combining some error correcting codes to reduce the coding rate to achieve better system performance by exploiting the additional information bit.

\section{Acknowledgements}

This work was supported by World Class University R32-2012-000-20014-0, BSRP 2010-0020942 NRF, Korea, MEST 2012-002521, NRF Korea, National Nature Science Foundation of China (61201249), and Natural Science Foundation of Guangdong Province (S201104 0004068), China.

\section{REFERENCES}

[1] S. Das, N. Al-Dhahir and A. Calderbank, "Novel FullDiversity High Rate STBC for 2 and 4 Transmit Antennas,” IEEE Communication Letters, Vol. 10, No. 3, 2006, pp.171-173. doi : 10.1109/LCOMM.2006.1603374

[2] V. Tarokh, N. Seshedri and A. R. Calderbank,
“Space-Time Codes for High Data Rate Wireless Communication: Performance Criterion and Code Construction," IEEE Transactions on Information Theory, Vol. 44, No. 2, 1998, pp. 744-765.doi : 10.1109/18.661517

[3] V. Tarokh and A. R. Calderbank, "Space-Time Block Codes from Orthogonal Designs,” IEEE Transactions on Information Theory, Vol. 45, No. 5, 1999, pp. 1456-1467.doi : 10.1109/18.771146

[4] S. M. Alamouti, “A Simple Transmit Diversity Technique for Wireless Communications,” IEEE Journal on Select Areas in Communications, Vol. 16, No. 8, 1998, pp.1451-1458.doi : 10.1109/49.730453

[5] B. Hassibi, B. M. Hochwald, A. Shokrollahi and W. Sweldens, "Representation Theory for High-rate Multiple- antenna Code Design,” IEEE Transations on Information Theory, Vol. 47, No. 9, 2001, pp. 2335-2367.

[6] H. Jafarkhani, "A Quasi-orthogonal Space Time Block Code,” IEEE Transactions on Communication, Vol. 49, No.1, 2001, pp. 1-4. doi : 10.1109/26.898239

[7] H. N. Duy, Na. H. Nguyen, T. Nguyen and D. Hoang, "High-Rate Space-Time Block Coding Schemes," IEICE Transactions on Fundamentals of Electronics, Communications and Computer Sciences, Vol. E91-A, No. 11, 2008, pp. 3393-3397. 